\title{
The moderating effect of gender on the relationship between coping and suicide risk in a Portuguese community sample of adults
}

\author{
Rui C. Campos ${ }^{1}$, Ronald R. Holden ${ }^{2}$, Fátima Costa ${ }^{1}$, Ana Rita Oliveira ${ }^{1}$, Marta Abreu ${ }^{1}$, and Natália Fresca ${ }^{1}$ \\ ${ }^{1}$ Departamento de Psicologia, Escola de Ciências Sociais, Universidade de Évora, Évora, Portugal and ${ }^{2}$ Department of Psychology, Queen's \\ University, Kingston, Ontario, Canada
}

\begin{abstract}
Background and aims(s): The study evaluated the contribution of coping strategies, based on the Toulousiane conceptualization of coping, to the prediction of suicide risk and tested the moderating effect of gender, controlling for depressive symptoms.

Method: A two-time data collection design was used. A community sample of 195 adults (91 men and 104 women) ranging in age from 19 to 65 years and living in several Portuguese regions, mostly in Alentejo, participated in this research.

Results: Gender, depressive symptoms, control, and withdrawal and conversion significantly predicted suicide risk and gender interacted with control, withdrawal and conversion, and social distraction in the prediction of suicide risk. Coping predicted suicide risk only for women. Conclusions: Results have important implications for assessment and intervention with suicide at-risk individuals. In particular,the evaluation and development of coping skills is indicated as a goal for therapists having suicide at-risk women as clients.
\end{abstract}

\author{
Keywords \\ coping, gender, interaction, suicide risk, \\ community-based sample
}

\section{History}

Received 9 April 2016

Accepted 27 May 2016

Published online 13 September 2016

\section{Introduction}

Globally, suicide is one of the primary causes of death claiming the lives of approximately one million persons annually (National Institute of Mental Health, 2009). For every death by suicide, it is estimated that there are 18 times as many attempted suicides (Moscicki, 1997). Even more common is suicide ideation and it is considered to be present in $2.3-14.6 \%$ of the general population (Casey et al., 2006). In Portugal, more than a thousand people die by suicide every year (10.3 per 100000 in the population), with suicide being the leading cause of unnatural death (National Institute of Statistics, 2013). Collectively, death by suicide, attempted death by suicide, and suicide ideation constitute a significant public health issue in all countries of the world.

\section{Gender and suicide risk}

Biological sex is an identified risk factor for both suicide and attempted suicide with women more likely than men to attempt suicide and men more likely than women to die by suicide (e.g. Overholser et al., 2012). This inversion of relative risk for biological sex between attempted and completed suicide has been termed the gender paradox (Canetto \& Sakinofsky, 1998). Although varying somewhat in degree, this inversion appears to generally exist across countries and times. Previously thought to be an exception

Correspondence: Rui C. Campos, Departamento de Psicologia, Escola de Ciências Sociais, Universidade de Évora, Apartado 94, Évora 7002554, Portugal. Tel: + 351 969046400. Fax: + 351266768073. E-mail: rcampos@uevora.pt to this paradox, recent data from China (Zhang et al., 2014) also now indicate that that country's male completed suicide rate currently exceeds the female rate. Because biological sex is a stable risk factor for suicide and the gender paradox occurs as a consistent phenomenon within suicidal behavior, biological sex becomes an important construct to recognize in suicide prediction and intervention. Although it should be noted that biological sex may be a proxy for other related construct(s) (e.g. sex roles and related individual differences) that underlie these sex differences (Canetto \& Cleary, 2012; Witte et al., 2012), biological sex is a relatively simply measured, but fallible, indicator for summarizing gender differences.

\section{Coping and gender}

Generally, coping can be defined as the efforts that individuals take to manage psychological stress (Lazarus, 1993), or as a set of cognitive and behavioral efforts used by individuals to deal with internal and external requirements in stress situations that are evaluated as exceeding personal resources. Coping involves the mobilization of cognitive and behavioral efforts to manage, reduce, and minimize the internal and external demands that arise in an individual's interactions with the environment (Lazarus \& Folkman, 1984).

During the 1990s, the Social Psychology and Health Development Research Group at the University of Toulouse, France, under the guidance of Pierre Tap conducted research on the construction of personal and professional identity. In their approach, stressors and coping were incorporated within a perspective that included developmental processes and a 